\title{
Comparison of Different Algorithms of Approximation by Extensional Fuzzy Subsets
}

Gabriel Mattioli and Jordi Recasens

\begin{abstract}
How to approximate an arbitrary fuzzy subset by an adequate extensional one is a key question within the theory of Extensional Fuzzy Subsets. In a recent paper by the authors [19] different methods were provided to find good approximations. In this work these methods are compared in order to understand better the performance and improvement they give.
\end{abstract}

\section{Introduction}

Indistinguishability operators were introduced to fuzzify the concept of crisp equivalence relations. These operators allow to model the idea of "similarity" between elements, which is key to understand how we "identify" objects. The operation of identification is the mainstone to simplify the representation we have of the environment and understand the information given by our perception. Being able to identify objects enables us to store less quantity of information if favour of being able to extract a qualitative analysis of it.

An eye without a mechanism to identify objects is nothing but a sensor of outern reality. An eye with this mechanism becomes a perceptive system that can "understand" the environment.

Under an indistinguishability operator the observable fuzzy sets are the extensional ones. These sets correspond to the fuzzification of classical equivalence classes. Within the theory of Fuzzy Logic the first researcher to point the relevance of these sets was Zadeh when he discussed the concept of granularity [24].

Department of Mathematics and Computer Science

ETS Arquitectura del Valles

Universitat Politècnica de Catalunya

C/ Pere Serra 1-15. 08190 Sant Cugat del Valls. Spain

e-mail: gabriel.mattioli@upc.edu

e-mail: j.recasens@upc.edu 
If we assume that indistinguishability operators are a good model to understand similarities between objects (and there is evidence to think so), then a very interesting problem is how an arbitrary fuzzy subset can be approximated by an extensional one with the minimum loss of accuracy.

In a previous work by the authors [19] this problem was faced and 3 methods were derived for Archimedean t-norms ( $T=$ Łukasievicz and $T=\Pi$ product) and one for the Minimum t-norm.

Restricting to Archimedean t-norms, the first method was based on finding an adequate mean between two operators that provide the best upper and lower approximation by extensional fuzzy subsets of a given fuzzy subset $\mu$. The second one computed an adequate power of the lower approximation of $\mu$, and the last one found the solution solving a Quadratic Programming problem.

Big differences can be found between the first two and the last method. The QPbased one guarantees that the solution found is optimal while the first two do not. On the other hand, the last method suffers drastically the curse of dimensionality and becomes computationally unaffordable for large cardinalities of the universe of discourse $X$. The first two do not have this problem and work even when $X$ is non-finite.

The aim of this work is to compare in depth the mean-based and the power-based methods. In order to reduce the scope of this comparison we will restrict to the Łukasievicz t-norm and to finite sets. This has been done because in [19] explicit formulas were provided to find the best approximations when $T=€$, while the best approximation for $T=\Pi$ had to be found by numerical methods.

The work is structured as follows:

In Section 2 the Preliminaries to this work are given. In this section the definition and main properties of indistinguishability operators and extensional sets will be recalled.

Section 3 will show how the mean-based method can be built. Natural weighted means will be introduced first and explicit formulas will be provided to find the extensional fuzzy subset that better approximates $\mu$ following this method.

In Section 4 the power-based method will be given. First of all it will be shown how powers can be defined with respect to a t-norm $T$ and further how this can be used to find good approximations by extensional fuzzy subsets.

In Section 5 a comparison between these two methods will be provided. Fixed an indistinguishability operator $E$ we will study the output and error committed by each of the methods when approximating different fuzzy subsets and some conclusions will be extracted.

Finally, the Concluding Remarks of this work will be given in Section 6. 


\section{Preliminaries}

In this section the main concepts and results used in this work will be given. The definition of indistinguishability operator will be recalled as well as the main properties of the extensional fuzzy subsets related to an indistinguishability operator.

First of all let us recall the well known Ling's Theorem which introduces the concept of additive generator $t$ of a continuous Archimedean t-norm. Additive generators will prove to be very useful further in this work.

Theorem 1 [15] A continuous t-norm $T$ is Archimedean if and only if there exists a continuous and strictly decreasing function $t:[0,1] \rightarrow[0, \infty]$ with $t(1)=0$ such that

$$
T(x, y)=t^{[-1]}(t(x)+t(y))
$$

where $t^{[-1]}$ is the pseudo inverse of $t$ defined by

$$
t^{[-1]}(x)= \begin{cases}1 & \text { if } x \leq 0 \\ t^{-1}(x) & \text { if } 0 \leq x \leq t(0) \\ 0 & \text { if } t(0) \leq x\end{cases}
$$

The function $t$ will be called an additive generator of the $t$-norm and two generators of the t-norm $T$ differ only by a positive multiplicative constant.

If $T=€$ is the Łukasievicz t-norm, then an additive generator is $t(x)=1-x$.

If $T=\Pi$ is the Product t-norm, then $t(x)=-\log (x)$.

Definition 1 Let $T$ be a t-norm.

- The residuation $\vec{T}$ of $T$ is defined for all $x, y \in[0,1]$ by

$$
\vec{T}(x \mid y)=\sup \{\alpha \in[0,1] \mid T(\alpha, x) \leq y\}
$$

- The birresiduation $\overleftrightarrow{T}$ of $T$ is defined for all $x, y \in[0,1]$ by

$$
\overleftrightarrow{T}(x, y)=\min \{\vec{T}(x \mid y), \vec{T}(y \mid x)\}=T(\vec{T}(x \mid y), \vec{T}(y \mid x))
$$

When the t-norm $T$ is continuous Archimedean, these operations can be rewritten in terms of the additive generator $t$.

Proposition 1 Let $T$ be a continuous Archimedean t-norm generated by an additive generatort. Then:

- $T(x, y)=t^{[-1]}(t(x)+t(y))$

- $\vec{T}(x \mid y)=t^{[-1]}(t(y)-t(x))$

- $\overleftrightarrow{T}(x, y)=t^{[-1]}(|t(x)-t(y)|)$ 
Indistinguishability operators are the fuzzification of classical equivalence relations and model the intuitive idea of "similarity" between objects. For a more detailed explanation on this operators readers are referred to [4], [21].

Definition 2 Let $T$ be a t-norm. A fuzzy relation $E$ on a set $X$ is a $T$-indistinguishability operator if and only if for all $x, y, z \in X$

a) $E(x, x)=1$ (Reflexivity)

b) $E(x, y)=E(y, x)$ (Symmetry)

c) $T(E(x, y), E(y, z)) \leq E(x, z)(T$-transitivity).

Whereas indistinguishability operators represent the fuzzification of equivalence relations, extensional fuzzy subsets play the role of fuzzy equivalence classes altogether with their intersections and unions. Extensional fuzzy subsets are a key concept in the comprehension of the universe of discourse $X$ under the effect of an indistinguishability operator $E$ as they correspond with the observable sets or granules of $X$.

Definition 3 Let $X$ be a set and $E$ a T-indistinguishability operator on X. A fuzzy subset $\mu$ of $X$ is called extensional with respect to $E$ if and only if:

$$
\forall x, y \in X \quad T(E(x, y), \mu(y)) \leq \mu(x) .
$$

We will denote $H_{E}$ the set of all extensional fuzzy subsets of $X$ with respect to $E$.

Extensional fuzzy subsets have been widely studied in the literature [7], [11], [12].

If the t-norm $T$ is continuous Archimedean then the condition of extensionality can be rewritten in terms of additive generators. This result will be recalled several times along this paper.

Lemma 1 Let $E$ be a $T$-indistinguishability operator on a set $X . \mu \in H_{E}$ if and only if $\forall x, y \in X$ :

$$
t(E(x, y))+t(\mu(y)) \geq t(\mu(x)) .
$$

Proof.

$$
\begin{aligned}
& \mu \in H_{E} \Leftrightarrow T(E(x, y), \mu(y)) \leq \mu(x) \\
\Leftrightarrow & t^{-1}(t(E(x, y))+t(\mu(y))) \leq \mu(x) .
\end{aligned}
$$

And as $t$ is a monotone decreasing function this is equivalent to

$$
t(E(x, y))+t(\mu(y)) \geq t(\mu(x)) .
$$

\section{Approximation using Means}

In this section we will propose a method to approximate an arbitrary fuzzy subset by an extensional one. First we will introduce two approximation operators, $\phi_{E}(\mu)$ 
and $\psi_{E}(\mu)$, that provide the best upper and lower approximation respectively by extensional fuzzy subsets of $\mu$ given an indistinguishability operator $E$. The method will consist in computing an adequate weight in order to minimize an error function between $\mu$ and the natural weighted mean of $\phi_{E}(\mu)$ and $\psi_{E}(\mu)$.

Definition 4 Let $X$ be a set and $E$ a $T$-indistinguishability operator on $X$. The maps $\phi_{E}:[0,1]^{X} \rightarrow[0,1]^{X}$ and $\psi_{E}:[0,1]^{X} \rightarrow[0,1]^{X}$ are defined $\forall x \in X$ by:

$$
\begin{gathered}
\phi_{E}(\mu)(x)=\sup _{y \in X} T(E(x, y), \mu(y)), \\
\psi_{E}(\mu)(x)=\inf _{y \in X} \vec{T}(E(x, y) \mid \mu(y)) .
\end{gathered}
$$

$\phi_{E}(\mu)$ is the smallest extensional fuzzy subset greater than or equal to $\mu$; hence it is its best upper approximation by extensional fuzzy subsets. Analogously, $\psi_{E}(\mu)$ provides the best approximation by extensional fuzzy subsets smaller than or equal to $\mu$. From a topological viewpoint these operators can be seen as closure and interior operators on the set $[0,1]^{X}[11]$. It is remarkable that these operators also appear in a natural way in fields such as fuzzy rough sets [20], fuzzy modal logic [6], [5], fuzzy mathematical morphology [8] and fuzzy contexts [3] among many others.

Though $\phi_{E}(\mu)$ and $\psi_{E}(\mu)$ provide extensional fuzzy subsets that approximate $\mu$ there is no guarantee in general that there are no better approximations of $\mu$ by extensional fuzzy subsets. In [19] the authors faced this problem and provided three methods to find approximations for Archimedean t-norms and one for the Minimum t-norm. The two methods compared in this paper were introduced there.

Definition 5 [1] Let $t:[0,1] \rightarrow[-\infty, \infty]$ be a non-increasing monotonic map, $x, y \in$ $[0,1]$ and $r \in[0,1]$. The weighted quasi-arithmetic mean $m_{t}$ of $x$ and $y$ is defined as:

$$
m_{t}^{r}(x, y)=t^{-1}(r \cdot t(x)+(1-r) \cdot t(y))
$$

$m_{t}$ is continuous if and only if $\{-\infty, \infty\} \nsubseteq \operatorname{Ran}(t)$.

There is a bijection between the set of continuous Archimedean t-norms and the set of quasi-arithmetic means by taking as map the additive generator $t$ of the t-norm [14]. Under this interpretation in the literature quasi-arithmetic means are sometimes called natural means [17], as we will recall them from now on.

We want to approximate $\mu$ by $m_{t}^{r}\left(\phi_{E}(\mu), \psi_{E}(\mu)\right)$. Below we prove that this mean is extensional for any value of $r$.

Proposition 2 [19] Let $X$ be a set and $\mu, v$ extensional fuzzy subsets of $X$ with respect to an indistinguishability operator $E$ on $X$. Then:

$$
m^{r}(\mu, v) \in H_{E} .
$$

Corollary 1 Let $\mu$ be a fuzzy subset on a set $X$ and $E$ an indistinguishability operator. Then:

$$
m^{r}\left(\phi_{E}(\mu), \psi_{E}(\mu)\right) \in H_{E}
$$


It is straightforward that for the limit values $r=0,1$ this mean is equal to $\phi_{E}(\mu)$ and $\psi_{E}(\mu)$ respectively. The question that arises here is for what value of $r$ the error made in this approximation is the lowest one. In mathematical terms, this problem reduces to finding the minimum value of the following function:

$$
F(r)=\left\|\mu-m^{r}\left(\phi_{E}(\mu), \psi_{E}(\mu)\right)\right\|
$$

Considering the Euclidean distance, without loss of generalization, minimizing the previous expression is equivalent to minimize the square of the norm.

$$
F(r)=\left\|\mu-m^{r}\left(\phi_{E}, \psi_{E}\right)\right\|^{2}
$$

For the Łukasievicz t-norm the result below provides an explicit formula to find this optimal weight $r$.

In order to simplify the notation we will denote $\mu_{i}=\mu\left(x_{i}\right), \phi_{i}=\phi_{E}(\mu)\left(x_{i}\right)$ and $\psi_{i}=\psi_{E}(\mu)\left(x_{i}\right)$.

Theorem 2 [19] Let $\mu$ be a fuzzy subset of a finite set $X=\left\{x_{1}, \ldots, x_{n}\right\}$ and $T=t$ the Eukasiewicz t-norm. Then the expression $F(r)=\left\|\mu-m^{r}\left(\phi_{E}(\mu), \psi_{E}(\mu)\right)\right\|^{2}$ is minimized when:

$$
r=\frac{\sum \mu_{i} \cdot \phi_{i}-\sum \mu_{i} \cdot \psi_{i}-\sum \phi_{i} \cdot \psi_{i}+\sum \psi_{i}^{2}}{\sum \phi_{i}^{2}+\sum \psi_{i}^{2}-2 \sum \phi_{i} \cdot \psi_{i}}
$$

\section{Approximation using Powers}

In this section we will provide another method to find an approximation of an arbitrary fuzzy subset $\mu$ by an extensional one. This method will be based on approximating $\mu$ by an adequate power $\psi_{E}(\mu)^{r}$ of its lower approximation operator with respect to the t-norm. It will be shown how, for values $r<1$, the fuzzy subset $\psi_{E}(\mu)^{r}$ is extensional and that a global minimum of the error made can be obtained.

Let us recall the definition of power with respect to a t-norm $T$.

Definition 6 Let $T$ be a t-norm and $n$ a natural number. We will call the $n^{\text {th }}$ power of $X$ with respect to $T$ to:

$$
T^{n}(x)=T(\overbrace{x, x, \ldots, x}^{n}) .
$$

To simplify notation we will denote $T^{n}(x)=x^{n}$.

It is possible to extend this definition to all positive rational numbers as follows.

Definition 7 Let $T$ be a t-norm and $n$ a natural number. We will define $x$ to the power of $1 / n$ with respect to $T$ as: 


$$
x^{1 / n}=\sup _{z \in[0,1]}\left\{T^{n}(z) \leq x\right\}
$$

and for $p, q$ natural numbers,

$$
x^{p / q}(x)=\left(x^{1 / q}\right)^{p}
$$

Passing to the limit it is possible to define $x^{r}$ for all $r \in \mathbb{R}^{+}$for continuous $\mathrm{t}$ norms.

The following result allows us to calculate powers by using an additive generator $t$ of $T$.

Proposition 3 Let $T$ be an Archimedean t-norm with additive generator $t$ and $r \in$ $\mathbb{R}^{+}$. Then:

$$
x^{r}=t^{[-1]}(r \cdot t(x))
$$

It is straightforward to observe from the previous proposition that $r \leq s \Rightarrow x^{r} \geq x^{s}$. Besides, the continuity of $t$ assures continuity of powers when we let the exponent vary.

The key idea of this method follows from the next Corollary 2.

Proposition 4 [19] Let $E$ be an indistinguishability operator of a set $X, \mu$ an extensional fuzzy subset of $E$ and $r \leq 1$. Then

$$
\mu^{r} \in H_{E}
$$

Corollary $2 \psi(\mu)^{r}$ is extensional for $r \leq 1$.

The problem of approximating a fuzzy subset $\mu$ by an adequate power $\psi(\mu)^{r}$ reduces then to compute the value of $r$ for which the following function is minimized.

$$
F(r)=\left\|\mu-\psi_{E}(\mu)^{r}\right\|^{2}
$$

For the Łukasievicz t-norm we have the following result.

As it was done in the previous section we will denote $\mu_{i}=\mu\left(x_{i}\right), \phi_{i}=\phi_{E}(\mu)\left(x_{i}\right)$ and $\psi_{i}=\psi_{E}(\mu)\left(x_{i}\right)$.

Theorem 3 [19] Let $\mu$ be a fuzzy subset of a finite set $X=\left\{x_{1}, \ldots, x_{n}\right\}$ and $T=t$ the Łukasiewicz t-norm. Then $F(r)=\left\|\mu-\psi_{E}(\mu)^{r}\right\|^{2}$ is minimized when

$$
r=\frac{\sum \mu_{i}+\sum \psi_{i}-\sum \mu_{i} \cdot \psi_{i}-n}{2 \sum \psi_{i}-\sum \psi_{i}^{2}-n}
$$

It would be expectable to find an analogous method to find good approximations of $\mu$ by extensional fuzzy subsets using powers of $\phi_{E}$. In [19] it is discussed and illustrated with a counterexample that this is not possible in general. 


\section{Comparative Analysis of the Two Methods Proposed}

In this section we will compare the two methods proposed in this paper to approximate arbitrary fuzzy subsets by extensional ones with respect to a given indistinguishability operator. We will compare the output and error committed by each of the methods applied to four fuzzy subsets of a finite set $X$.

Let us consider the following $Ł$-indistinguishability operator

$$
E=\left(\begin{array}{llllll}
1 & 0.9 & 0.7 & 0.4 & 0.2 \\
0.9 & 1 & 0.7 & 0.4 & 0.2 \\
0.7 & 0.7 & 1 & 0.4 & 0.2 \\
0.4 & 0.4 & 0.4 & 1 & 0.2 \\
0.2 & 0.2 & 0.2 & 0.2 & 1
\end{array}\right)
$$

and the fuzzy subsets

$$
\mu_{1}=\left(\begin{array}{l}
0.9 \\
0.5 \\
0.6 \\
0.8 \\
0.3
\end{array}\right) \mu_{2}=\left(\begin{array}{l}
0.2 \\
0 \\
0.2 \\
0.6 \\
0.9
\end{array}\right) \mu_{3}=\left(\begin{array}{l}
0.7 \\
0.5 \\
0.1 \\
0.8 \\
0.6
\end{array}\right) \mu_{4}=\left(\begin{array}{l}
0.1 \\
0.9 \\
0.2 \\
0.8 \\
0.5
\end{array}\right) .
$$

It is straightforward to observe that none of these sets is extensional with respect to $E$.

The corresponding upper and lower approximations by extensional fuzzy subsets of these sets are

$$
\begin{gathered}
\phi\left(\mu_{1}\right)=\left(\begin{array}{l}
0.9 \\
0.8 \\
0.6 \\
0.8 \\
0.3
\end{array}\right) \phi\left(\mu_{2}\right)=\left(\begin{array}{l}
0.2 \\
0.1 \\
0.2 \\
0.6 \\
0.9
\end{array}\right) \phi\left(\mu_{3}\right)=\left(\begin{array}{l}
0.7 \\
0.6 \\
0.4 \\
0.8 \\
0.6
\end{array}\right) \phi\left(\mu_{4}\right)=\left(\begin{array}{l}
0.8 \\
0.9 \\
0.6 \\
0.8 \\
0.5
\end{array}\right) . \\
\psi\left(\mu_{1}\right)=\left(\begin{array}{l}
0.6 \\
0.5 \\
0.6 \\
0.8 \\
0.3
\end{array}\right) \psi\left(\mu_{2}\right)=\left(\begin{array}{l}
0.1 \\
0 \\
0.2 \\
0.6 \\
0.8
\end{array}\right) \quad \psi\left(\mu_{3}\right)=\left(\begin{array}{l}
0.4 \\
0.4 \\
0.1 \\
0.7 \\
0.6
\end{array}\right) \psi\left(\mu_{4}\right)=\left(\begin{array}{l}
0.1 \\
0.2 \\
0.2 \\
0.7 \\
0.5
\end{array}\right) .
\end{gathered}
$$

Let us denote $m_{i}$ and $p_{i}$ the fuzzy extensional subsets obtained following the mean-based and power-based methods respectively for each of the sets $\mu_{i}(i=$ $1, \ldots, 4)$. The output fuzzy extensional subsets are given are given below. 


$$
\begin{aligned}
& m_{1}=\left(\begin{array}{l}
0.75 \\
0.65 \\
0.6 \\
0.8 \\
0.3
\end{array}\right) m_{2}=\left(\begin{array}{l}
0.1667 \\
0.0667 \\
0.2 \\
0.6 \\
0.8667
\end{array}\right) m_{3}=\left(\begin{array}{l}
0.5565 \\
0.5043 \\
0.2565 \\
0.7522 \\
0.6
\end{array}\right) m_{4}=\left(\begin{array}{l}
0.4043 \\
0.5043 \\
0.3739 \\
0.7434 \\
0.5
\end{array}\right) . \\
& p_{1}=\left(\begin{array}{l}
0.6436 \\
0.5545 \\
0.6436 \\
0.8218 \\
0.3763
\end{array}\right) p_{2}=\left(\begin{array}{l}
0.1374 \\
0.0415 \\
0.2332 \\
0.6166 \\
0.8083
\end{array}\right) \quad p_{3}=\left(\begin{array}{l}
0.4910 \\
0.4910 \\
0.2365 \\
0.7455 \\
0.6606
\end{array}\right) p_{4}=\left(\begin{array}{l}
0.3185 \\
0.3942 \\
0.3942 \\
0.7728 \\
0.6214
\end{array}\right) .
\end{aligned}
$$

Table 1 shows the error committed by each of these approximations. The error has been computed taking the euclidean distance between the original fuzzy subset and the extensional approximation of it.

\begin{tabular}{|c||c|c|c|c|}
\hline & $\mu_{1}$ & $\mu_{2}$ & $\mu_{3}$ & $\mu_{4}$ \\
\hline$\phi_{i}$ & $\mathrm{e}=0.3$ & $\mathrm{e}=0.1$ & $\mathrm{e}=0.3162$ & 0.8062 \\
\hline$\psi_{i}$ & $\mathrm{e}=0.3$ & $\mathrm{e}=0.1414$ & $\mathrm{e}=0.3317$ & 0.7071 \\
\hline$m_{i}$ & $\mathrm{e}=0.2121$ & $\mathrm{e}=0.0816$ & $\mathrm{e}=0.2177$ & $\mathrm{e}=0.5316$ \\
\hline$p_{i}$ & $\mathrm{e}=0.2773$ & $\mathrm{e}=0.1243$ & $\mathrm{e}=0.2627$ & $\mathrm{e}=0.5973$ \\
\hline
\end{tabular}

Table 1 Table comparing the error committed by the different methods proposed for $\mu_{1}, \mu_{2}, \mu_{3}$ and $\mu_{4}$

Let us analyze now the results obtained for both methods.

A first consideration about the sets $\mu_{1}, \mu_{2}, \mu_{3}$ and $\mu_{4}$ is that they show different levels of variance and "distance" from extensionality. It can be observed for instance that the upper and lower approximation of $\mu_{2}$ is very similar to the initial set while the approximations of $\mu_{4}$ commit a much bigger error.

Another interesting previous consideration is that for $\mu_{2}$ and $\mu_{3}, \phi_{E}\left(\mu_{i}\right)$ commits a slightly lower error than $\psi\left(\mu_{i}\right)$ whereas the error committed in the approximation of $\mu_{4}$ is lower in the case of $\psi_{E}\left(\mu_{4}\right)$. From here we can infer that there is no guarantee that the approximation provided by $\phi_{E}$ is better than the one given by $\psi_{E}$ and viceversa

Comparing the results obtained with the two new methods proposed in the approximation of the given sets we observe that in all cases the approximation using the mean-based or the power-based method improves the approximation made either by the $\phi_{E}$ and $\psi_{E}$ operators.

Finally it can be observed that in all cases the approximation made using the mean-based method is better than the one given by the power-based one.

According to the results obtained, the conclusion of this analysis is that there is evidence to consider that the two methods proposed improve the ones existing in the literature. However, it is a must to recall that the analysis made is not exhaustive 
and similar analysis should be done with other indistinguishabilities operators and extensional fuzzy subsets in order to assert this improvement formally.

\section{Concluding Remarks}

In this work we have recalled two of the methods proposed by the authors in [19] to approximate arbitrary fuzzy subsets by extensional ones for $€$-indistinguishability operators. Both methods are built upon two operators that provide the smallest extensional subset that contains $\mu\left(\phi_{E}(\mu)\right)$ and the biggest extensional one contained in $\mu\left(\psi_{E}(\mu)\right)$.

The first method consists in computing an adequate mean of the upper and the lower approximation of $\mu$.

The second one is based on finding an adequate power (homotecy) for which $\psi(\mu)^{r}$ is a better extensional approximation of $\mu$.

In both cases explicit formulas have been given to compute the best approximations following the methods.

Finally, both methods have been tested for a given indisitinguishability $E$ and 4 extensional sets. Despite a deeper comparison should be done to extract conclusive assertions, the results obtained show relevant evidence that the two methods proposed improve the approximation given by $\phi_{E}(\mu)$ and $\psi_{E}(\mu)$. Among them, the results obtained suggest that the mean-based method is slightly better than the power-based one.

Acknowledgements Research partially supported by project number TIN2009-07235.

The first author is supported by the Universitat Politècnica de Catalunya (UPC).

\section{References}

1. Aczél, J.: Lectures on functional equations and their applications. Academic Press, NYLondon (1966)

2. M.S. Bazaraa, H.D. Sherali, C.M. Shetty. Nonlinear programming: theory and algorithms 2nd ed. John Wiley \& Sons. New York. (1993)

3. Bělohlávek, R.: Fuzzy Relational Systems: Foundations and Principles. Kluwer Academic Publishers, New York (2002)

4. Boixader, D., Jacas, J., Recasens, J.: Fuzzy equivalence relations: advanced material. In: Dubois, D., Prade, H. (eds.) Fundamentals of Fuzzy Sets, pp. 261-290. Kluwer Academic Publishers, New York (2000)

5. Boixader, D., Jacas, J., Recasens, J.: Upper and lower approximation of fuzzy sets. Int. J. of General Systems 29, 555-568 (2000)

6. Bou, F., Esteva, F., Godo, L., Rodríguez, R.: On the Minimum Many-Valued Modal Logic over a Finite Residuated Lattice. Journal of Logic and computation (2010) doi:10.1093/logcom/exp062

7. Castro, J.L., Klawonn, F.: Similarity in Fuzzy Reasoning. Mathware \& Soft Computing 2, 197-228 (1996) 
8. J. Elorza, R. Fuentes-González, J. Bragard, P. Burillo: On The Relation Between Fuzzy Preorders, Fuzzy Closing Morphological Operators And Fuzzy Consequence Operators. Proceedings of ESTYLF2010 Conference. Huelva. 133-138 (2010)

9. Flanders, H.: Differentiation under the integral sign. American Mathematical Monthly 80 (6): 615-627 (1973)

10. Garmendia L., Recasens, J.: How to make T-transitive a proximity relation. IEEE Transactions on Fuzzy Systems, vol 17, issue 1, 200-207 (2009)

11. Jacas, J., Recasens, J.: Fixed points and generators of fuzzy relations. J. Math. Anal. Appl $186,21-29$ (1994)

12. Jacas, J., Recasens, J.: Fuzzy T-transitive relations: eigenvectors and generators. Fuzzy Sets and Systems 72, 147-154 (1995)

13. Jacas, J., Recasens, J. Maps and Isometries between indistinguishability operators. Soft Computing 6. 14-20. (2002)

14. Jacas, J., Recasens, J.: Aggregation of T-Transitive Relations. Int J. of Intelligent Systems 18, 1193-1214 (2003)

15. Klement, E.P., Mesiar, R., Pap E.: Triangular norms. Kluwer Academic Publishers, Dordrecht (2000)

16. Mattioli, G., Recasens, J. Dualities and Isomorphisms between Indistinguishabilities and Related Concepts FUZZ'IEEE Taiwan, pp 2369 - 2374 . (2011).

17. Mattioli, G., Recasens, J. Natural Means of Indistinguishability Operators. Advances in Computational Intelligence Communications in Computer and Information Science Vol 299, pp 261-270. IPMU2012 Catania (2012).

18. Mattioli, G., Recasens, J. Structural Analysis of Indistinguishability Operators and Related Concepts. Informations Sciences (submitted)

19. Mattioli, G., Recasens, J. Approximating Arbitrary Fuzzy Subsets by Extensional Ones. IEEE Transactions on Fuzzy Systems (submitted)

20. Morsi, N.N., Yakout, M.M.: Axiomatics for fuzzy rough sets. Fuzzy Sets and Systems 100, 327-342 (1998)

21. Recasens, J. Indistinguishability Operators. Modelling Fuzzy Equalities and Fuzzy Equivalence Relations Series: Studies in Fuzziness and Soft Computing, Vol. 260 (2011)

22. Valverde, L.: On the Structure of F-indistinguishability Operators. Fuzzy Sets and Systems 17, 313-328 (1985)

23. Williamson, J.H.: Lebesgue Integration . Holt, Rinehart and Winston (1962)

24. Zadeh. L.A. Similarity relations and fuzzy orderings. Inform. Sci. 3, 177-200 (1971)

25. Zadeh, L.A.: Fuzzy sets and information granularity. In: Gupta, M.M., Ragade, R.K., Yager, R.R. (eds.) Advances in Fuzzy Set Theory and Applications, pp. 3-18. Amsterdam, NorthHolland (1979)

26. Zadeh, L.A.: Toward a theory of fuzzy information granulation and its centrality in human reasoning and fuzzy logic. Fuzzy Sets and Systems 90, 111-127 (1997) 